\title{
Similaridade da qualidade das águas subterrâneas da bacia do Rio Negro, MS
}

\author{
Similarity of groundwater quality of the Rio Negro watershed, MS
}

\author{
Amaury de Souza ${ }^{* 1}$, Giancarlo Lastoria ${ }^{2}$, Sandra Gabas ${ }^{3}$, \\ Sávio de Brito Fontenele ${ }^{4}$, Ana Paula Garcia Oliveira ${ }^{5}$ \\ 1, 2, 3Universidade Federal de Mato Grosso do Sul, Campo Grande, Brasil \\ ${ }^{4}$ Universidade Federal do Ceará, Fortaleza, Brasil
}

\begin{abstract}
Resumo
Para investigar a similaridade, em escala espacial e temporal, da qualidade das águas da micro-bacia do pantanal do rio Negro, empregou-se a análise de agrupamento e análise fatorial. As amostras simples de água foram coletadas em 12 pontos (georreferenciados) no ano de 2004. Foram avaliados os seguintes parâmetros: $\mathrm{Ca}^{+2}, \mathrm{Mg}^{+2}, \mathrm{Na}^{+1}+\mathrm{K}$ ${ }^{+1}, \mathrm{HCO}_{3}^{-1}, \mathrm{Cl}^{-1}, \mathrm{SO}_{4}^{-2}, \mathrm{pH}, \mathrm{As}^{+3}, \mathrm{Cd}^{+2}, \mathrm{Cr}^{+6}, \mathrm{Cu}^{+2}, \mathrm{Fe}_{3}^{+3}, \mathrm{Mn}^{+2}, \mathrm{Mo}^{+6}, \mathrm{~Pb}^{+2}$, Alcalinidade, Dureza, Resíduo seco, C.E, $\mathrm{PO}_{4}^{-3}, \mathrm{NO}_{3}^{-1}, \mathrm{NO}_{2}^{-1}$. A redução dos dados pela análise por componentes principais resultou em três componentes, com valores próprios, os quais explicam até $92,4 \%$ da variância total, sendo a primeira componente com $48,6 \%$, a segunda componente com $23,6 \%$, e a terceira componente com 20,2\%. A variabilidade das componentes pode ser usada para indicar o processo hidrodinâmico que controla a qualidade das águas, sem perda de características significativas. A análise de agrupamento hierárquico formou três grupos distintos, sendo determinantes os tipos e concentrações dos sais analisados. A qualidade de água da microbacia é influenciada, principalmente, por dois fatores: fontes pontuais de poluentes e a sazonalidade climática.
\end{abstract}

Palavras-chave: Análise de agrupamento. Fontes de poluição. Sazonalidade climática.

\begin{abstract}
To investigate the similarity in spatial and temporal scale of water quality of the Black River wetland micro-basin we used cluster analysis and factor analysis. Simple water samples were collected at 12 points (georeferenced) in 2004. The following parameters were evaluated: $\mathrm{Ca}^{+2}, \mathrm{Mg}^{+2}, \mathrm{Na}{ }^{+1}+\mathrm{K}^{+1}, \mathrm{HCO}_{3}^{-1}, \mathrm{Cl}^{-1}, \mathrm{SO}_{4}^{-2}, \mathrm{pH}, \mathrm{As}^{+3}, \mathrm{Cd}^{+2}$, $\mathrm{Cr}^{+6}, \mathrm{Cu}^{+2}, \mathrm{Fe}_{3}^{+3}, \mathrm{Mn}^{+2}, \mathrm{Mo}^{+6}, \mathrm{~Pb}^{+2}$, alkalinity, hardness , dry residue, C.E, $\mathrm{PO}_{4}^{-3}, \mathrm{NO}_{3}^{-1}, \mathrm{NO}_{2}^{-1}$. Data reduction by principal component analysis yielded three components with eigenvalues, which explain up to $92.4 \%$ of total variance, with the first component with $48.6 \%$, the second component with $23.6 \%$, the third component with $20.2 \%$. The variability of the components can be used to indicate the hydrodynamic process that controls the quality of water without loss of significant features. The hierarchical cluster analysis formed three distinct groups, determining the types and concentrations of salts analyzed. The water quality of the watershed is mainly influenced by two factors: point sources of pollutants and climatic seasonality.
\end{abstract}

Keywords: Cluster analysis. Sources of pollution. Climatic seasonality. 


\section{Introdução}

$\mathrm{O}$ fornecimento de água de qualidade para diversos fins, como uso doméstico, irrigação, serviços e indústria, é o principal objetivo de qualquer projeto de recursos hídricos. Assim, faz-se necessária a proteção dos recursos hídricos devido a sua importância no contexto socioeconômico. O estudo da qualidade das águas ganhou importância para compreender processos e fatores (naturais ou antropogênicos) que contribuem para a sua degradação.

As atividades de pesquisa e desenvolvimento tecnológico, relacionadas aos recursos hídricos no pantanal sul-mato-grossense, enfrentam dificuldades para manter um monitoramento contínuo de parâmetros de qualidade e quantidade dos recursos hídricos. Vale destacar que esses problemas se tornam maiores quando se trata de águas subterrâneas. Esse monitoramento teria importância fundamental na geração de um banco de dados regional bem da situação dos poços de extração tornando-se uma ferramenta para gerenciamento da qualidade, identificação de fontes de poluição e de vulnerabilidade.

O mapeamento de aquíferos e fontes de poluição, o diagnóstico das águas subterrâneas e dos pontos de vulnerabilidade resultantes da integração água $x$ atividade humana, apontando a sua relação com resultados da contaminação dos corpos hídricos, podem ser vistos em diversos estudos recentes (SALGADO, E. V. et al., 2011; ALMEIDA et al., 2010; FURQUIM et al., 2010; FERNANDES, F. B. P. et al., 2010; BARBIÉRO et al. (2008, 2002); ZHOU et al., 2007; ANDRADE et al., 2007; ÁVILA et al., 2007; STIGTER et al., 2006; RAO, 2006; DONADIO et al., 2005; ROMERO et al., 2004; PAUWELS et al., 2001 e SAKAMOTTO et al., 1996). Tais ações são fundamentais na gestão eficiente dos recursos hídricos.

No monitoramento da qualidade das águas analisase parâmetros relacionados à cor, sólidos totais, alcalinidade, dureza, nitrogênio, fósforo, matéria orgânica, metais pesados, dentre outros. Quando esses parâmetros são analisados separadamente, o entendimento de suas inter-relações se torna mais difícil, podendo interferir negativamente no processo de tomada de decisão do planejamento dos recursos hídricos. No entanto, a utilização de técnicas estatísticas de análise fatorial em componentes principais possibilita reduzir o número de parâmetros a serem monitorados e conhecer suas relações. Além disso, através de uma análise de agrupamento ou "cluster" é possível identificar as variáveis responsáveis pela dispersão das observações e evidenciar possíveis agrupamentos, de acordo com seu grau de similaridade. Em recursos hídricos, GUPTA, et al., 2005; RAO et al., 2006; BOYACIOGLU, 2006; MADHUMITHA DAS, 2009; J. K. PATHAK, 2008 e LIU et al., 2003 utilizaram-nas para avaliar impactos ambientais e/ou identificar variáveis de maior significância no conjunto das variáveis que definem a qualidade das águas.

A ferramenta de agrupamento hierárquico é uma técnica de classificação, dentro das análises estatísticas multivariadas, que tem sido utilizada individualmente ou em conjunto com outras técnicas para bancos de dados com tamanho limitado (McNEILA et al., 2005). Esta ferramenta tem como princípio a identificação das características dos objetos pelas similaridades dos parâmetros processados. Pelos resultados obtidos em diversos estudos, a mesma se apresenta como uma eficiente ferramenta em estudos na qualidade da água (ANDRADE et al., 2005 e PALÁCIO et al., 2009).

Neste sentido, aplicou-se a ferramenta do agrupamento hierárquico da estatística de análise multivariada, objetivando investigar a sua aplicabilidade como um instrumento de monitoramento e suporte à gestão da qualidade da água subterrânea em bacias hidrográficas do pantanal sul-mato-grossense.

\section{Material e métodos}

\subsection{Descrição da área}

A sub-bacia do rio Negro está localizada na região central do Estado de Mato Grosso do Sul, entre as coordenadas geográficas $18^{\circ} 00^{\prime} 00^{\prime \prime}$ e $20^{\circ} 30^{\prime} 00^{\prime \prime}$ de latitude Sul e $54^{\circ} 30^{\prime} 00^{\prime \prime}$ e $57^{\circ} 30^{\prime} 00^{\prime \prime}$ de longitude Oeste (Figura 1). Sua área de drenagem é de $34.948 \mathrm{~km}^{2}$ e seu principal constituinte é o próprio rio Negro (527 km de extensão), (PERH, MS, 2010). A rede hidrográfica é formada pelos

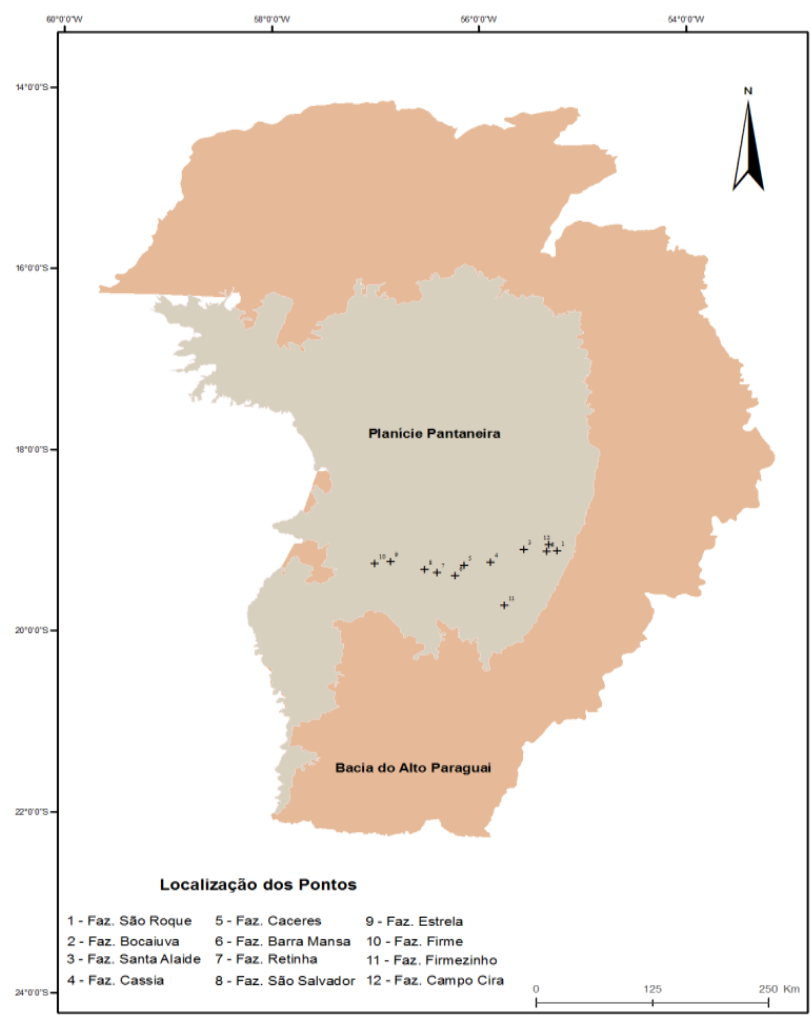

Figura 1: Mapa de Localização do Pantanal MatoGrossense na Bacia do Alto Paraguai. 
Tabela 1. Localização, coordenadas geográficas dos pontos de coletas de dados dos sítios do Rio Negro.

$\begin{array}{llcccc} & \text { Local } & \text { Latitude } & \text { Longitude } & \text { UTM_E } & \text { UTM_N } \\ 1 & \text { Faz. São Roque } & -19121800.00 & -55244300.00 & 684,675,224 & 7884768480.00 \\ 2 & \text { Faz. Campo Cira } & -19055270.00 & -55327000.00 & 676,044,669 & 7892217707.00 \\ 3 & \text { Faz. Bocaiuva } & -19126800.00 & -55346900.00 & 673,875,456 & 7884320272.00 \\ 4 & \text { Faz St }{ }^{\mathrm{a}} \text { Alaide } & -19108468.00 & -55564518.00 & 650,998,675 & 7886551393.00 \\ 5 & \text { Faz. Cássia } & -19246063.00 & -55890942.00 & 616,561,364 & 7871573926.00 \\ 6 & \text { Faz. Cáceres } & -19284569.00 & -56143318.00 & 590,013,965 & 7867462840.00 \\ 7 & \text { Faz. Barra Mansa } & -19398500.00 & -56232300.00 & 580,607,944 & 7854899078.00 \\ 8 & \text { Faz. Retinha } & -19359500.00 & -56407400.00 & 562,236,792 & 7859287195.00 \\ 9 & \text { Faz. São Salvador } & -19331300.00 & -56527100.00 & 549,673,785 & 7862446415.00 \\ 10 & \text { Faz. Estrela } & -19240557.00 & -56856100.00 & 515,123,575 & 7872548795.00 \\ 11 & \text { Faz.Firme } & -19264300.00 & -57004500.00 & 499,527,128 & 7869927911.00 \\ 12 & \text { Faz. Firmezinho } & -19725818.00 & -55756248.00 & 630,334,678 & 7818381669.00\end{array}$

rios Negro, do Peixe, Negrinho, Criminoso e Taboco, pelos córregos do Garimpo, Anhuma, Acampamento, Branco, Mimoso e Baguaçu, pelas vazantes do Castelo, do Brejão, do Grande, do Mangabal, de Santa Clara e da Alegria e pelo corixo Vermelho (PERH-MS, 2010).

O clima da região pode ser caracterizado como tropical, megatérmico, com precipitação anual de 1.182,5 $\mathrm{mm}$, com duas estações bem definidas: período chuvoso, de novembro a março (quando ocorre cerca de $72 \%$ da precipitação anual); período seco, que vai de abril a outubro (SORIANO \& ALVES, 2003). A temperatura média anual é de $25,5^{\circ} \mathrm{C}$, oscilando entre $20,7^{\circ} \mathrm{C}$ e $28,0^{\circ} \mathrm{C}$. A umidade relativa do ar média anual é $82 \%$, com pouca variação ao longo do ano, e a insolação anual registrada é de cerca de 2.348 horas, sendo julho o mês com valores mais elevados de incidência de luz solar.

\section{Geologia da sub bacia do pantanal}

A Bacia do Pantanal é delimitada à leste e à nordeste pelos metassedimentos do Grupo Cuiabá e rochas graníticas, os quais se encontram recobertos por sedimentos paleozóicos da bacia do Paraná. Ao norte e ao sul seus limites são os metassedimentos da Faixa Paraguai. As serras de Amolar e de Aguapé, que fazem parte do cráton Amazônico/Rio Apa, delimitam, respectivamente, as bordas oeste e noroeste da bacia. Na parte central da bacia, a formação basal é constituída das areias finas e grossas com fragmentos de rochas carbonáticas provenientes de sequências plataformais da faixa Paraguai. Sobreposto a esta unidade, aflora um pacote arenoso de granulométrica média a grossa, com quantidade significante de fragmentos de arenito ferruginoso. A porção mais superior é constituída por sedimentos arenosos, siltico-argilosos, argilo-arenosos e areno-conglomeráticos, semi-consolidados a inconsolidados. Todo o pacote sedimentar é depositado discordantemente sobre os metassedimentos da Faixa Paraguai e sobre o Cráton Amazônico (Almeida, 1964 e 1965).
A economia da região está ligada quase que totalmente à pecuária. As indústrias que predominam são do ramo de laticínios e matadouros. No que diz respeito ao abastecimento de água, os municípios da área (Rio Negro, Rio Verde de Mato Grosso, São Gabriel do Oeste, Corguinho e Aquidauana) são supridos por água de poços artesianos. Somente Aquidauana usa o sistema misto, rio e poço.

\subsection{Análise das águas subterrâneas}

Para caracterizar as águas subterrâneas da bacia, coletou-se amostras de água em poços tubulares durante o ano de 2004. As análises foram realizadas nos laboratórios de Análises Químicas da Escola de Química da Universidade Federal Fluminense para as variáveis: $\left(\mathrm{Ca}^{+2}\right),\left(\mathrm{Mg}^{+2}\right), \mathrm{Na}^{+1}+\mathrm{K}^{+1}, \mathrm{HCO}_{3}^{-1}, \mathrm{Cl}^{-1}, \mathrm{SO}_{4}^{-2}, \mathrm{pH}, \mathrm{As}^{+3}, \mathrm{Cd}^{+2}$, $\mathrm{Cr}^{+6}, \mathrm{Cu}^{+2}, \mathrm{Fe}_{3}^{+3}, \mathrm{Mn}^{+2}, \mathrm{Mo}^{+6}, \mathrm{~Pb}^{+2}, \mathrm{CE}, \mathrm{PO}_{4}^{-3}, \mathrm{NO}_{3}^{-1}, \mathrm{NO}_{2}^{-1}$, Alcalinidade, Dureza e Resíduo seco.

Para agrupar os poços que possuem concentrações similares dos constituintes iônicos presentes nas amostras, empregou-se a análise da estatística multivariada, conhecida como técnica de agrupamento hierárquico. O processamento das informações foi efetuado pelo software SPSS, versão 16.0. Antes do processamento, buscou-se eliminar o efeito de escala e unidades com a conversão das variáveis para escores padrões ( $Z$ escore). $\mathrm{O}$ algoritmo de agrupamento empregado foi o ward's method pela sua grande eficiência em combinar um pequeno número de amostras com um mesmo número de observações e a distância de similaridade empregada foi a Euclidiana ao quadrado. O resultado deste procedimento é apresentado por meio de um dendrograma. O ponto de corte do dendrograma, para definir o número de grupos, fundamentou-se na técnica apresentada por CORRAR et al. (2009) em que o corte ocorre no momento em que a distância reescalonada do coeficiente de aglomeração apresenta a maior variação. Os grupos formados pela 
Tabela 2- Relação dos parâmetros analisados pelo Laboratório da U.F.F. 2004.

\begin{tabular}{|c|c|c|}
\hline Parâmetro (símbolo) & Unidade & Método utilizado para análise \\
\hline Calcio (Ca) & $\mathrm{mg} / \mathrm{l}$ & ICP/AES \\
\hline Magnésio (Mg) & $\mathrm{mg} / \mathrm{l}$ & ICP/AES \\
\hline Potássio(K) & $\mathrm{mg} / \mathrm{l}$ & ICP/AES \\
\hline Sódio (Na) & $\mathrm{mg} / \mathrm{l}$ & ICP/AES \\
\hline Bicarbonato $\left(\mathrm{HCO}_{3}\right)$ & $\mathrm{mg} / \mathrm{l}$ & Titulação Potenciométrica \\
\hline Cloro $(\mathrm{Cl})$ & $\mathrm{mg} / \mathrm{l}$ & Método Argentimétrico \\
\hline Sulfato $\left(\mathrm{SO}_{4}\right)$ & $\mathrm{mg} / \mathrm{l}$ & Cromatografia iônica \\
\hline Cond. Elétrica a $25^{\circ} \mathrm{C}$ & $\mu \mathrm{S}$ & Condutivimetro \\
\hline Resíduo Seco $110^{\circ} \mathrm{C}$ & $\mathrm{mg} / \mathrm{l}$ & Gravimétrico \\
\hline Dureza Total em $\mathrm{CaCO}_{3}$ & $\mathrm{mg} / \mathrm{l}$ & Titulação/EDTA \\
\hline Alcalinidade Total & $\mathrm{mg} / \mathrm{l}$ & Método Potenciomérico \\
\hline $\mathrm{pH}$ & $X X X X X$ & Potenciométrico \\
\hline $\mathrm{NO}_{3}$ & $\mathrm{mg} / \mathrm{l}$ & Método da Redução do Cádmio \\
\hline $\mathrm{NO}_{2}$ & $\mathrm{mg} / \mathrm{l}$ & Método da Redução do Cádmio \\
\hline \multirow{2}{*}{$\mathrm{PO}_{4}$} & \multirow{2}{*}{$\mathrm{mg} / \mathrm{l}$} & Método Colorimétrico com Ácido \\
\hline & & Ascórbico \\
\hline As & $\mathrm{mg} / \mathrm{l}$ & ICP/AES \\
\hline $\mathrm{Cd}$ & $\mathrm{mg} / \mathrm{l}$ & ICP/AES \\
\hline $\mathrm{Cr}$ & $\mathrm{mg} / \mathrm{l}$ & ICP/AES \\
\hline $\mathrm{Cu}$ & $\mathrm{mg} / \mathrm{l}$ & ICP/AES \\
\hline Fé & $\mathrm{mg} / \mathrm{l}$ & ICP/AES \\
\hline $\mathrm{Mn}$ & $\mathrm{mg} / \mathrm{l}$ & ICP/AES \\
\hline Mo & $\mathrm{mg} / \mathrm{l}$ & ICP/AES \\
\hline $\mathrm{Pb}$ & $\mathrm{mg}$ & ICP/AES \\
\hline $\mathrm{Al}$ & $\mathrm{mg}$ & ICP/AES \\
\hline $\mathrm{Si}$ & $\mathrm{mg}$ & ICP/AES \\
\hline
\end{tabular}

técnica do agrupamento tiveram suas médias comparadas pelo teste t de Student aos níveis de 5\% e 1\%, ambos após a execução da análise de variância.

\section{Resultados e discussão}

A partir das informações das Tabelas 3 e 4, do cruzamento com as características geológicas da área e da Figura 2, é possível formatar um quadro de características da água subterrânea no pantanal mato-grossense.

A presença do cátion sódio como dominante na classificação das águas subterrâneas da sub-bacia do Rio Negro se dá devido à presença de determinadas argilas, principalmente as do grupo das montmorilonitas sódicas, identificadas na região e que, sob condições favoráveis, pode incrementar processos de troca catiônica envolvendo o $\mathrm{Na}^{+1}$. A única exceção é na região oeste, próximo à foz do Rio Negro, onde o cátion maior é o $\mathrm{Ca}^{+2}$, proveniente de águas que percolam calcários do grupo Corumbá. Em contrapartida, o ânion dominante é o $\mathrm{HCO}_{3}^{-1}$, proveniente do $\mathrm{CO}_{2}$ da atmosfera e dos solos e/ou da dissolução de rochas carbonáticas do Grupo Corumbá. Foi registrada a presença de $\mathrm{Cr}^{6+}$ com valores oscilando de 0,0025 mg/l na Fazenda Retinha (sítio 8) a 0,0042 mg/l na Fazenda São Roque (sítio 1). No Rio Negro também foi detectada uma concentração de 0.005 $\mathrm{mg} / \mathrm{l}$ de $\mathrm{Cr}^{6+}$. Esse Cr6+ provavelmente é originário de atividades industriais que despejam seus resíduos na parte alta da bacia. $\mathrm{O} \mathrm{Cu}^{+2}$ no âmbito das analises realizadas varia de $0,001 \mathrm{mg} / \mathrm{l}$, na Fazenda São Salvadoro (sítio 9), a 0,042 mg/l, na Fazenda Cáceres (sítio 6). A presença de $\mathrm{Cu}^{+2}$ é devido à utilização desse elemento na composição dos agrotóxicos de caráter fungífero, em 
Tabela 3- Carga fatorial para a variável qualidade de águas subterrâneas da bacia hidrográfica do rio Negro.

\begin{tabular}{crrrc}
\hline Variável & \multicolumn{1}{c}{ Fator1 } & \multicolumn{1}{c}{ Fator2 } & Fator3 & Communality \\
\hline $\mathrm{Ca}^{+2}$ & $\mathbf{0 , 7 6}$ & 0,45 & $-0,02$ & 0,79 \\
$\mathrm{Mg}^{+2}$ & $\mathbf{0 , 9 2}$ & $-0,02$ & 0,02 & 0,85 \\
$\mathrm{Na}^{+1}+\mathrm{K}^{+1}$ & $\mathbf{0 , 9 2}$ & 0,30 & 0,19 & 0,97 \\
$\mathrm{HCO}^{-1}$ & $\mathbf{0 , 8 9}$ & $-0,40$ & 0,15 & 0,98 \\
$\mathrm{Cl}^{-1}$ & 0,15 & $\mathbf{0 , 9 0}$ & 0,30 & 0,92 \\
$\mathrm{SO}^{-2}$ & $\mathbf{0 , 8 9}$ & 0,13 & 0,13 & 0,83 \\
$\mathrm{pH}^{-2}$ & 0,62 & $-0,20$ & $\mathbf{0 , 7 0}$ & 0,91 \\
$\mathrm{C} . \mathrm{E}$ & $\mathbf{0 , 8 8}$ & 0,25 & 0,36 & 0,97 \\
$\mathrm{NO}_{3}^{-1}$ & 0,07 & $\mathbf{0 , 9 9}$ & 0,02 & 0,98 \\
$\mathrm{Na}^{+1}$ & $\mathbf{0 , 9 6}$ & 0,09 & 0,06 & 0,94 \\
\hline $\mathrm{Var}(\%)$ & 0,54 & 0,23 & 0,14 & 0,92 \\
\hline
\end{tabular}

utilização nos perímetros agrícolas, na parte alta da bacia. $\mathrm{O} \mathrm{Fe}{ }^{+3}$ se apresenta com teor variando de 0,001 a 0,3 $\mathrm{mg} / \mathrm{l}$, exceto na área adjacente, a Fazenda Campo Cira (sítio 2) com 0,52 mg/l e na Fazenda Bocaiúva (sítio 3) com 6,1 mg/l. Próximo a essa região o poço perfurado, no qual os primeiros $240 \mathrm{~m}$ foram expressos por uma areia fina a grosseira, localmente conglomerática com grande quantidade de seixos de quartzo e cimentação ferruginosa é de se prever que o ferro, detectado naquela compartimentação de amostragem, seja proveniente da supramencionada cimentação. $\mathrm{O} \mathrm{Mn}^{+2}$ no universo das análises químicas apresenta um teor inexpressivo.

$\mathrm{Na}$ análise foi registrada a presença de teor elevado de $\mathrm{PO}_{4}^{-3}$ em todos os pontos amostrados. Na Fazenda Firmezinho (sítio 12) o valor encontrado foi de 2900,0 $\mathrm{mg} / \mathrm{l}$. Problema idêntico ocorre com relação aos fertilizantes com base em compostos nitrogenados, razão pela qual ocorrem teores elevados de $\mathrm{NO}_{3}^{-1} \mathrm{e} \mathrm{NO}_{2}^{-1}$ nos pontos amostrados. Outro dado relevante nessa amostragem é $\mathrm{o} \mathrm{As}^{+3}$ presente em todos os pontos de amostragem com teor superior a $0,01 \mathrm{mg} / \mathrm{l}$. Esse $\mathrm{As}^{+3}$ tem sua origem nos herbicidas, fungicidas e carrapaticidas pertencentes ao grupo dos pesticidas inorgânicos (BRAILLE, 1979). A presença de metais pesados e de princípios ativos dos agrotóxicos, detectados nos sedimentos do Pantanal do Rio Negro, provavelmente é função da alta capacidade do sedimento de funcionar como um excelente sumidouro dos supramencionados poluentes. Ainda nesta abrangência, conclui-se que esses poluentes poderão ser disponibilizados para o meio ambiente, via coluna de água, devido às atividades dos organismos bentônicos presentes nesses sedimentos (CUSTODIO \&LAMAS, 1963).

Foram aplicadas técnicas de análise fatorial em componentes principais e de agrupamento, ao conjunto de 25 parâmetros de qualidade de águas, correspondendo às fontes subterrâneas analisadas na sub-bacia do rio Negro no ano de 2004. A partir da análise de correlação aplicada aos parâmetros, observou-se que a maioria desses estão fortemente correlacionados, com alta significância $(\mathrm{P}<0,0001)$.

A análise fatorial em componentes principais aplicada aos dados condensou as variáveis analisadas em três fatores ordenados, explicando 91,8\% da variância total, sendo o Fator 1 (F1) responsável por 54,4\% dessa variância. As variáveis com maiores cargas fatoriais, nesse fator, foram $\mathrm{Ca}^{+2}, \mathrm{Mg}^{+2}, \mathrm{Na}^{+1}+\mathrm{K}^{+1}, \mathrm{HCO}^{-1}, \mathrm{SO}^{-2}, \mathrm{CE}$ e $\mathrm{Na}^{+1}$, embora as demais variáveis também apresentem forte relação com esse fator, dados os elevados valores das cargas fatoriais e das comunalidades finais, sendo que essas representam o quanto da variância da variável é reproduzida pelos fatores comuns (Tabela 3). O Fator 2 (F2) respondeu por 23,0\% da variância total e incluiu variáveis com altos valores de comunalidades finais, como $\mathrm{Cl}^{-1}$ e $\mathrm{NO}^{-1}$, enquanto que o Fator 3 (F3) esteve influenciado pelo $\mathrm{pH}$. Elementos esses dos fatores F2 e F3 relacionados aos nutrientes presentes nas águas.

O Fator 1 é fortemente relacionado com a salinidade das águas. De acordo com ANDRADE (1989), são representativas aquelas variáveis cujas cargas fatoriais são as mais elevadas e devem sempre ser superiores a 0,30 (Tabela 3). Em função das maiores cargas fatoriais das variáveis que compõem os três fatores nos períodos, as 10 variáveis de qualidade das águas podem ser representadas por: Fator 1 - componente de salinidade.

$O$ resultado do processamento das análises das amostras de água dos poços pode ser visualizado no dendrograma (Figura 2). O ponto de corte da distância reescalonada do coeficiente da aglomeração, para definição dos grupos, ocorreu ao valor calculado entre às distâncias de 5,96 e 7,98. Pelo ponto de corte formou-se três grupos, sendo o grupo 1 composto por 3 poços, o grupo 2 por 5 poços e o grupo 3 por 4 poços. Observa-se pelo dendrograma que a maior similaridade foi registrada entre os poços que compõem o grupo 1 , sendo o segundo e o terceiro os mais heterogêneos.

A similaridade entre os grupos definidos pelo ponto de corte no dendrograma pode ser mais bem analisada e interpretada através da Tabela 3.

A partir da formação dos grupos, procurou-se, ainda, investigar qual ou quais dos atributos da qualidade dos poços que compõem o grupo 1 apresentaram os menores valores das concentrações médias dos atributos à exceção dos atributos da Alcalinidade Total e do Cálcio, os quais não se mostraram significativos na separação dos grupos. Da mesma forma, o grupo 2 apresentou os valores médios intermediários das concentrações dos referidos atributos e o grupo 3, notoriamente com as maiores médias das concentrações dos atributos.

De acordo com os resultados obtidos, pode-se observar que não ocorreram significativas variações na sua classificação. Isso pode ocorrer devido às características das águas subterrâneas estarem fortemente relacionadas com as formações geológicas da bacia em estudo. Além disso, os resultados sugerem um monitoramento com 


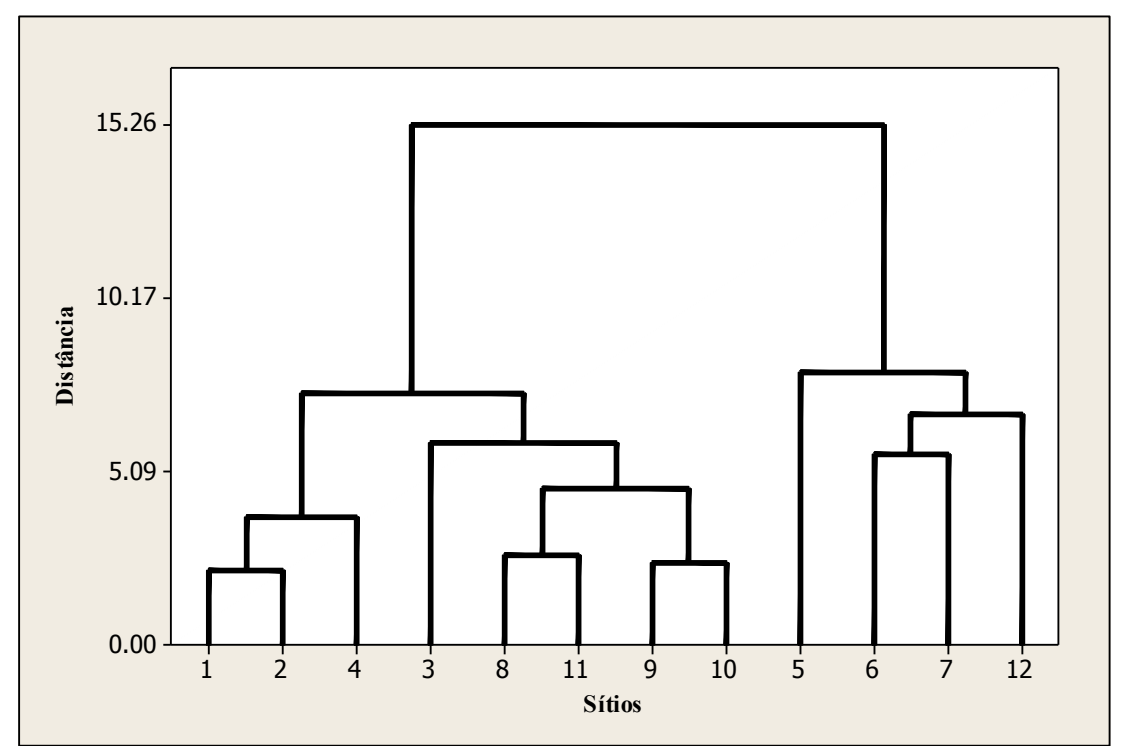

Figura 2 - Similaridade das concentrações iônicas das águas dos poços localizados na bacia do rio Negro, MS.

Tabela 4 - Média, desvio padrão, valores máximo e mínimo dos atributos investigados nas águas dos poços da bacia do rio Negro, MS, definidos pela análise de agrupamento.

\begin{tabular}{|c|c|c|c|c|c|c|c|c|c|c|c|c|}
\hline \multirow{2}{*}{ Parâmetros } & \multicolumn{4}{|c|}{ Grupo 1} & \multicolumn{4}{|c|}{ Grupo 2} & \multicolumn{4}{|c|}{ Grupo 3} \\
\hline & Média & $\mathrm{dp}$ & Mín. & Máx. & Média & $\mathrm{dp}$ & Mín. & Máx. & Média & $d p$ & Mín. & Máx. \\
\hline${ }^{* *} \mathrm{Ca}^{+2}$ & 2.73 & 3.63 & 0.29 & 6.90 & 4.30 & 2.54 & 1.80 & 7.50 & 16.35 & 7.13 & 6.40 & 22,00 \\
\hline${ }^{* *} \mathrm{Mg}^{+2}$ & 0.79 & 0.80 & 0.20 & 1.70 & 3.20 & 1.54 & 1.90 & 5.70 & 6.77 & 2.83 & 3.50 & 10,00 \\
\hline${ }^{* *} \mathrm{Na}+1+\mathrm{K}^{+1}$ & 9,00 & 5.29 & 3,00 & 13,00 & 7.02 & 2.73 & 3,00 & 10,00 & 45.50 & 19.12 & 31,00 & 72,00 \\
\hline${ }^{* *} \mathrm{HCO} 3{ }^{-1}$ & 22.86 & 8.19 & 13.72 & 29.54 & 91.78 & 34.97 & 42.20 & 126.60 & 165.36 & 116.89 & 40.09 & 314.40 \\
\hline${ }^{*} \mathrm{Cl}^{-1}$ & 20.17 & 17.34 & 7.90 & 40,00 & 5.38 & 4.93 & 0.40 & 13.60 & 28.40 & 21.79 & 7.40 & 55.80 \\
\hline $\mathrm{SO}_{4}^{-2}$ & 4.27 & 4.63 & 1.30 & 9.60 & 4.96 & 4.18 & 1.40 & 11.20 & 21.55 & 16.07 & 8.70 & 44.90 \\
\hline $\mathrm{pH}$ & 6.33 & 0.40 & 5.90 & 6.70 & 6.44 & 0.23 & 6.20 & 6.70 & 7.45 & 0.79 & 6.30 & 8.10 \\
\hline $\mathrm{As}^{+3}$ & 0.03 & 0.01 & 0.02 & 0.04 & 0.06 & 0.02 & 0.03 & 0.08 & 0.06 & 0.01 & 0.05 & 0.07 \\
\hline $\mathrm{Cd}^{+2}$ & 0.00 & 0,00 & 0.00 & 0.00 & 0.00 & 0,00 & 0.00 & 0.00 & 0.00 & 0,00 & 0.00 & 0.00 \\
\hline $\mathrm{Cr}^{+6}$ & 0.00 & 0.00 & 0.00 & 0.00 & 0.00 & 0.00 & 0.00 & 0.00 & 0.00 & 0,00 & 0.00 & 0.00 \\
\hline $\mathrm{Cu}^{+2}$ & 0.00 & 0,00 & 0.00 & 0.00 & 0.00 & 0.00 & 0.00 & 0.00 & 0.00 & 0.00 & 0.00 & 0.00 \\
\hline $\mathrm{Fe}^{+3}$ & 0.19 & 0.29 & 0.02 & 0.52 & 1.35 & 2.66 & 0.02 & 6.10 & 0.04 & 0.07 & 0.00 & 0.15 \\
\hline $\mathrm{Mn}^{+2}$ & 0.03 & 0.02 & 0.01 & 0.04 & 0.15 & 0.12 & 0.01 & 0.27 & 0.10 & 0.12 & 0.00 & 0.22 \\
\hline $\mathrm{Mo}^{+6}$ & 0.00 & 0,00 & 0.00 & 0.00 & 0.00 & 0.00 & 0.00 & 0.00 & 0.00 & 0.00 & 0.00 & 0.00 \\
\hline Alcalinidade & 22.86 & 8.19 & 13.72 & 29.54 & 91.78 & 34.97 & 42.20 & 126.60 & 179.08 & 132.87 & 40.09 & 348.15 \\
\hline Dureza & 17,00 & 16.64 & 5,00 & 36,00 & 58.20 & 29.25 & 23,00 & 92,00 & 119.25 & 21.75 & 99,00 & 140,00 \\
\hline Res. seco & 176.67 & 98.15 & 120,00 & 290,00 & 202,00 & 50.49 & 140,00 & 270,00 & 411.75 & 73.25 & 310,00 & 470,00 \\
\hline${ }^{* *}$ C.E & 141.67 & 92.83 & 67.30 & 245.70 & 199.80 & 78.49 & 96.70 & 283.20 & 523,00 & 146.59 & 371.60 & 723.80 \\
\hline $\mathrm{PO}^{-3}$ & 9.83 & 6.30 & 4.80 & 16.90 & 21.56 & 22.00 & 3.20 & 57.90 & 1014.93 & 1365,00 & 4.20 & 2900,00 \\
\hline${ }^{* *} \mathrm{NO}^{-1}$ & 3.56 & 2.20 & 1.45 & 5.84 & 0.13 & 0.23 & 0.01 & 0.54 & 7.00 & 10.96 & 0.03 & 23.09 \\
\hline $\mathrm{NO}_{2}^{-1}$ & 6.78 & 7.66 & 1,00 & 15.47 & 1.24 & 0.86 & 0.45 & 2.54 & 6.82 & 10.81 & 0.22 & 22.98 \\
\hline $\mathrm{Na}^{+1}$ & 6.40 & 3.37 & 2.30 & 8.80 & 3.75 & 2.02 & 0.75 & 6.40 & 33.00 & 22.55 & 15.00 & 66.00 \\
\hline
\end{tabular}


baixa frequência de dados amostrais e foco nos poços mais representativos do ponto de vista de uso pela comunidade.

Através da Figura 3 observa-se que os poços componentes dos grupos 2 e 3 encontram-se exatamente inseridos nas áreas de predominância da agricultura e pecuária intensiva (BACHEGA et al., 2009). Esta localização pode estar influenciada pela ação destas atividades agropecuárias.

BACHEGA et al., 2009, fez um estudo considerando o percentual de área de pastagem entre 1985 e o ano de 2007: houve um aumento na área de pastagens na Bacia do Negro e uma diminuição das áreas de matas. No ano de 1985 , a área de mata era de $20 \%$ da total, enquanto em 2007 passou a ser de $15 \%$ da área total, indicando, assim, um desmatamento na área da bacia. Em relação à pastagem, sua área teve um aumento de $17 \%$ da área total. Outra cobertura do solo da Bacia do Negro é o Cerrado, que em 2007 representa 30\% da área total.

Determinar qual fator (relevo, solos, uso e ocupação e/ou clima) que mais contribuiu para o aumento dos atributos da qualidade da água fica extremamente difícil pela complexidade destas inter-relações em decorrência das características multivariadas.

No entanto, a estatística multivariada pelo emprego da técnica do agrupamento hierárquico demonstra a capacidade de processar analiticamente todas as informações dos resultados das análises laboratoriais, mostrando para cada poço o efeito resultante de todas estas contribuições. Esta capacidade em processar tais informações fortalece o uso desta ferramenta como um instrumento de trabalho preciso, dinâmico e de extrema rapidez no processamento das análises laboratoriais.

De acordo com a classificação hidroquimica dos grupos definidos como similares pela análise de agrupamento empregou-se o "diagrama de Piper".

O grupo 1 é abrangido pelos sítios 1, 2 e 4, no diagrama de Piper, Figura 4 constata-se presença majoritária de elementos alcalinos em relação aos alcalino-terrosos nos sítios 1 e 2 e de alcalinos terrosos no sítio 4 , bem como de ácidos fortes em relação a ácidos fracos em todos os pontos. $\mathrm{O}$ pH médio dessas águas é de 6,3, não ocorrendo nesse setor valores máximos e mínimos muito afastados da média.

Quanto ao grau de dureza essas águas podem ser consideradas como brandas. Nesse setor, em termos

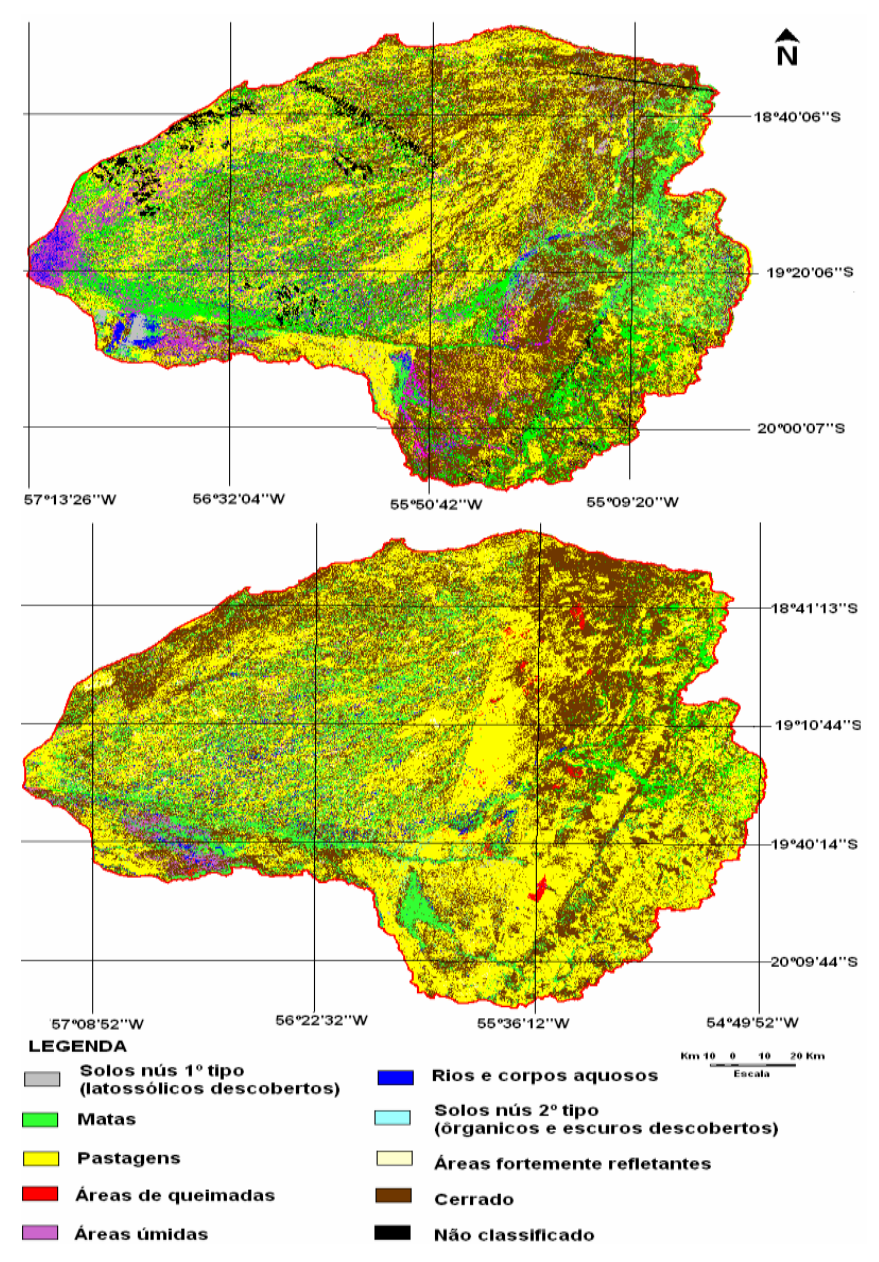

Figura 3. Mosaico das quatro classificações automáticas supervisionadas da imagem Landsat 5 (sensor TM) do ano de 1985 e 2007. Fonte-adaptada de BACHEGA et al, 2009. 

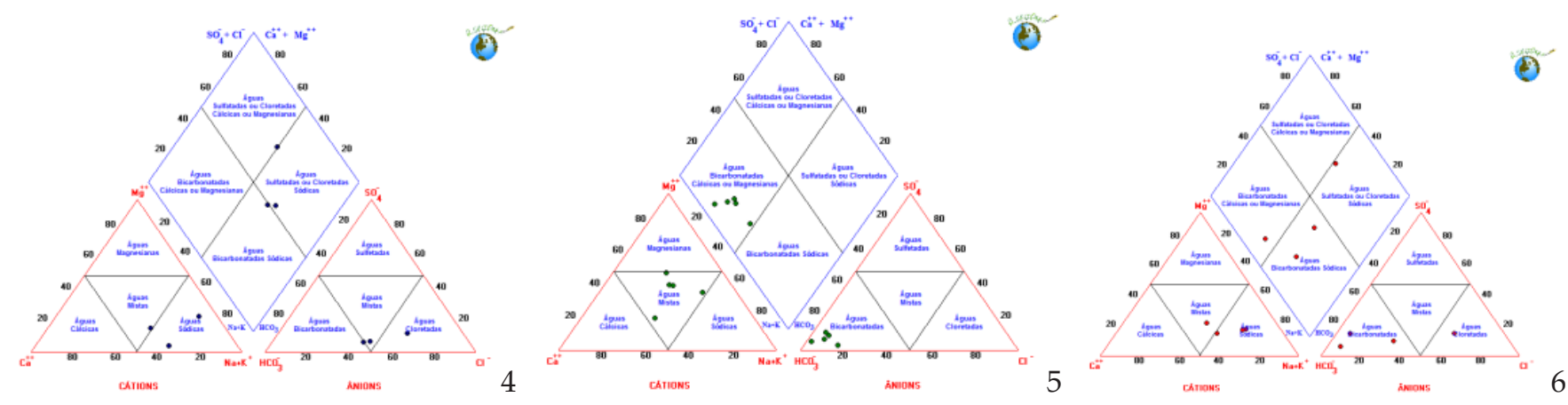

Figura 4- Grupo 1: Sítios 1, 2 e 4 - Fazendas São Roque, Campo Cira e St $t^{\mathrm{a}}$ Adelaide.Figura 5- Grupo 2: Sítios 3 , 8, 9, 10 e 11 - Fazendas Bocaiuva, Retinha, São Salvador, Estrela e Firme. Figura 6-Grupo 3: Sítios 5, 6, 7 E 12 Fazendas Cássia, Cáceres, Barra Mansa e Firmezinho.

de agressividade das águas, constata-se que as mesmas são essencialmente corrosivas. A classificação geral das águas pode ser considerada como $\mathrm{Na}^{+1}>\mathrm{Ca}^{+2}>\mathrm{Mg}^{+2} ; \mathrm{H}-$ $\mathrm{CO}_{3}^{-1}>\mathrm{Cl}^{-1}>\mathrm{SO}_{4}^{-2}$.

O grupo 2 é abrangido pelos sítios números 3, 8, 9,10 e 11. Essa área é caracterizada por exibir no gráfico de Stiff uma abrangência de águas do tipo bicarbonatadasódica. A única exceção é o ponto amostrado na Fazenda Cássia, que expõe uma água do tipo cloretada-mista, porém com tendência para bicarbonatada-mista. No diagrama de Piper verifica-se que os elementos alcalinos são mais presentes que os alcalino-terrosos, a exceção do sítio 3 ,caracterizado por ácidos fracos excederem ácidos fortes. O pH médio dessas águas é de 6,4 e os valores máximos e mínimos não se encontram, conforme já visto nas outras áreas, muito afastados da média. Em termos de dureza, segundo a classificação, a maioria das águas são um pouco duras (à exceção do sítio sete que é dura). Quanto à agressividade das águas constata-se que as mesmas são essencialmente corrosivas. A classificação geral das águas é $\mathrm{Na}^{+1}>\mathrm{Ca}^{+2}>\mathrm{Mg}^{+2}$ e $\mathrm{HCO}_{3}^{-1}>\mathrm{Cl}^{-1}>\mathrm{SO}_{4}^{-2}$.

O grupo 3 corresponde aos sítios 5, 6, 7 e 12. Nesse ponto de amostragem fica evidente no diagrama de Stiff que se está diante de uma água do tipo bicarbonatadasódica e no diagrama de Piper é constatado a ocorrência de elementos alcalinos sobrepujando os alcalino-terrosos, bem como de ácidos fracos excedendo os ácidos fortes. $\mathrm{O}$ pH é de 7,5 e a dureza pode ser correlacionada a uma água dura. Em termos de agressividade das águas constata-se que a mesma é essencialmente corrosiva. A classificação geral dessa água é $\mathrm{Na}^{+1}>\mathrm{Ca}^{+2}>\mathrm{Mg}^{+2}$ e $\mathrm{HCO}_{3}^{-1}>\mathrm{Cl}^{-1}>\mathrm{SO}_{4}^{-2}$.

A região dos sítios 10 e 11, seu traço marcante de área típica de água bicarbonatada-mista.

Neste estudo observou-se que não existe uma similaridade espacial contínua entre os poços, contudo identificou-se uma classificação distinta em cada grupo. Em estudos das águas subterrâneas da sub-bacia do rio Negro, foi identificado um predomínio de águas bicarbonatadas-mistas, independentes da sazonalidade climática.

\section{Conclusões}

A análise fatorial permitiu classificar as variáveis de maior significância na qualidade das águas, priorizando aquelas relacionadas com a salinidade, associada às características geológicas da bacia.

A análise de agrupamento classificou as fontes hídricas de acordo com a qualidade das águas, salinadades; porém, de modo geral, não ocorreram variações significativas nas de qualidade das águas nos períodos analisados, indicando que o monitoramento de qualidade das águas pode ser de baixa frequência. A utilização da estatística multivariada definiu os grupos heterogeneos, agrupando-os na forma de dendrograma, conforme a composição química de diferentes padrões de qualidades de água;

Os grupos formados pela análise de agrupamento são determinados a partir da formação hidrogeologia dos poços estudados;

Os íons $\mathrm{Ca}^{+2}, \mathrm{Mg}^{+2}, \mathrm{Na}^{+1}+\mathrm{K}^{+1}, \mathrm{HCO}^{-1}, \mathrm{SO}^{-2}$, $\mathrm{CE}$ e $\mathrm{Na}^{+1}$ são fortemente relacionados com a salinidade das águas, cujas cargas fatoriais são superiores a 0,544;

Apesar das características hidroquimicas dos três grupos diferirem estatisticamente a um nível de significância de $1 \%$, estes valores não superaram o limite aceitável de portabilidade destas águas.

Conclui-se que o comportamento dessas águas continua sendo de alcalinos excederem alcalino-terrosos, porém já se verifica uma tendência para os ácidos fortes terem uma supremacia em relação aos ácidos fracos. Constata-se, ainda, um predomínio de águas bicarbonatadas-mistas.

\section{Referências}

ALMEIDA, F.F.M.. Geologia do Centro-Oeste MatoGrossense. Bol. DGM, 215. Rio de Janeiro: Editorial DNPM, 133p,1964.

ALMEIDA, F.F.M.. Geologia da Serra da Bodoquena. Bol. DGM, 219. Rio de Janeiro: Editorial DNPM, 
$112 \mathrm{p}, 1965$.

ALMEIDA, T. I. R.; PARANHOS FILHO, A. C.; ROCHA, M. M.; SOUZA, G. F.; SÍGOLO, J. B.; BERTOLO, R. A. Estudo sobre as diferenças de altimetria do nível da água de lagoas salinas e hipossalinas no Pantanal da Nhecolândia: um indicativo de funcionamento do mega sistema lacustre. Geociências, v. 28, n. 4, p. 401-415, 2009.

\section{ALMEIDA, T.I.R. KARMANN, I.;PARANHOS} FILHO, A.C.;SÍGOLO , J. B.,BERTOLO, R.B. Os diferentes graus de isolamento da água subterrânea como origem de sua variabilidade: evidências isotópicas, hidroquímicas e da variação sazonal do nível da água no Pantanal da Nhecolândia. Geol. USP, Sér. cient. vol.10 no.3 São Paulo out. 2010.

ANDRADE, E.M. de; ARAÚJO, L. de F.P.; ROSA, M.F.; DISNEY, W.; ALVES, A.B. Seleção dos indicadores da qualidade das águas superficiais pelo emprego da análise multivariada. Engenharia Agrícola, Jaboticabal, v.27, n.3, p.683-690, set./dez. 2007.

ANDRADE, E. M. et al. Índice de qualidade de água, uma proposta para o vale do rio Trussu, Ceará. Revista Ciência Agronômica, v. 36, n. 2, p. 135-142, 2005.

ÁVILA, C.J.C.P.; ASSAD, E.D.; VERDESIO, J.J.; E.I.D, N.J.; SOARES, W.; FREITAS, M.A.V. DE. Geoprocessamento da informação hidrológica. ASFAGRO: trabalhos técnicos 2007.

Disponível em: <http://www.asfagro.org.br/trabalhos_ tecnicos $>$. Acesso em: 15 outubro. 2013.

BACHEGA, C. C. F.; PARANHOS FILHO, A. C.; TIVIROLI, V. A.; SOUZA MIRANDA, C. DE; ANACHE, J. A. Análise multitemporal da cobertura do solo na bacia do Rio Negro, MS. In: Simpósio Brasileiro de Sensoriamento Remoto, 14, 2009, Natal. Anais...São José dos Campos: INPE, 2009. p. 1253-1259. ISBN: 978-85-17-00044-7. Disponível na biblioteca digital URLib: < http://marte.dpi.inpe. $\mathrm{br} / \mathrm{col} / \mathrm{ltid}$. inpe.br/sbsr/.pdf $>$. Acesso em: setembro 2013.

BARBIÉRO, L.; QUEIROZ NETO, J. P.; CIORNEI, G., SAKAMOTO, A. Y.; CAPELLARI, B.; FERNANDES, E. Geochemistry of water and ground water in the Nhecolândia, Pantanal of Mato Grosso, Brazil: variability and associated processes. Wetlands, v. 22, p. 528"540, 2002.
BARBIÉRO, L.; REZENDE FILHO, A.; FURQUIM, S. A. C.; FURIAN; S. SAKAMOTO, A. Y. VALLES; GRAHAM, V. R. C.; FORT, M.; FERREIRA, R. P. D.; QUEIROZ NETO, J. P. Soil morphological control on saline and freshwater lake hydrogeochemistry in the Pantanal of Nhecolândia, Brazil. Geoderma, v. 148, p. 91-106, 2008.

BRANCO FILHO, H. C. DISTRIBUIÇÃO ESPACIAL E TEMPORAL DAS CARACTERÍSTICAS HIDROQUÍMICAS DAS ÁGUAS SUBTERRÂNEAS DO PANTANAL DO RIO NEGRO, 2005.

104 f. Dissertação de Mestrado em GeologiaUniversidade Federal do Rio de Janeiro. Rio de Janeiro, 2005.

BRAILLE, P.M et al. Manual de Tratamento de Águas Residuárias Industriais. São Paulo. Editorial Cetesb, 764p,(1979).

BOYACIOGLU H. "Surface water quality assessment using factor analysis", ISSN 0378-4738 = Water SA Vol. 32 No. 3,pp.389-394, 2006.

CORRAR, L. J.; PAULO, E.; FILHO, J. M. D. Análise Multivariada para os Cursos de Administração, Ciências Contábeis e Economia. 1. ed. 2. reimpr. São Paulo: Atlas, 541 p. 2009.

CUSTODIO, E. E LLAMAS M.E. Hidrologia Subterránea. Barcelona. Ediciones Omega, 1154p,(1983).

DONADIO, N.M.M.; GALBIATTI, J.A.; PAULA, R.C. DE. Qualidade da água de nascentes com diferentes usos do solo na bacia hidrográfica do Córrego Rico, São Paulo, Brasil. Engenharia Agrícola, Jaboticabal, v.25, n.1, p.115-125, 2005.

FERNANDES, F. B. P, ANDRADE, E. M. A. FONTENELE, S. B. , MEIRELES, A. C. M. , RIBEIRO, J. A. Análise de agrupamento como suporte à gestão qualitativa da água subterrânea no semiárido cearense. Revista Agro@mbiente On-line, v. 4, n. 2, p. 86-95, jul-dez, 2010

FURQUIM, S. A. C.; GRAHAM, R. C.; BARBIÉRO, L.; QUEIROZ NETO, J. P.; VIDAL-TORRADO, P. Soil mineral genesis and distribution in a saline lake landscape of the Pantanal Wetland, Brazil. Geoderma, v. 154, p. 518-528, 2010.

GUPTA,A. K, S. K. GUPTA AND PATIL. R.S, "Statistical analyses of coastal water quality for a port and harbour region in India". Environ. Monit. Asses. 102,pp.179-200 , 2005. 
KEHEW, A. E Applied Chemical Hydrogeology. New York: Prentice-Hall. 368 p. 2000.

LIU, CHEN-WUING, KAO-HUNG LIN, YI-MING KUO. "Application of factor analysis in the assessment of groundwater quality in a blackfoot disease area in Taiwan", The Science of the Total Environment 313, pp. 77-89, 2003.

MADHUMITHA DAS "Identifintificcation of effluent quality indicators for use in irrigation", journal of scientific and industrial resrech,Vol.68,pp 634-39, 2009.

McNEILA, V. H.; COXA, M. E.; MICAELA, P. Assessment of chemical water types and their spatial variation using multi-stage cluster analysis, Queensland - Australia. Journal of Hydrology. n. 310, p.181-200, 2005.

ONGLEY, E.D. Controle da poluição da água pelas atividades agrícolas. Tradução de: H.R.Gheyi; F.A.V.Damaceno; L.T.L. Brito. Campina Grande: UFPB, 2001. 92 p. (FAO, Irrigação e Drenagem, 55).

PALÁCIO, H. A. Q. et al. Salinidade da qualidade das águas superficiais da bacia do Curu, Ceará. Fortaleza-Ceará. Ciência Rural, v. 39, n. 9, p. 24942500, 2009.

PATHAK, J. K., Mohd Alam and Shikha Sharma, "Interpretation of Groundwater Quality Using Multivariate Statistical Technique in Moradabad City, Western Uttar Pradesh State, India", E-Journal of Chemistry Vol. 5, No.3,pp. 607-619, 2008.

PAUWELS, H.; LACHASSAGNE, P.C.; BORDENAVE, P.; FOUCHER, J.; MARTELAT, A. Temporal variability of nitrate concentration in a schist aquifer and transfer to surface waters. Applied Geochemistry, Amsterdam, v.16, n.6, p.583-596, 2001.

RAO, S.N. Groundwater potential index in a crystalline terrain using remote sensing data. Environmental Geology, Berlin, v.50, n.7, p.1.0671.076, 2006.

RAO, S.N., D. JOHN DEVADAS AND K. V.SRINIVASA RAO, "Interpretation of groundwater quality using principal component analysis from Anantapur district, Andhra Pradesh, India",Environmental Geosciences, v. 13, no.4, pp. 239-259, 2006.
ROMERO, A.H.H.; HERNÁNDEZ, C.T.; MALO, E.A.; MENDOZA, R.B. Water quality and presence of pesticides - southern México. Marine Pollution Bulletin, Oxford, v.48, n.11-12, p.1.130-1.141, 2004.

SALGADO, E. V., ANDRADE, E. M., FONTENELE, S. B., MEIRELES, A. C. M. SIMILARIDADE DAS VARIÁVEIS HIDROQUÍMICAS COM O USO DA ANÁLISE MULTIVARIADA, NA BACIA DO SALGADO, CEARÁ. Revista Caatinga, Mossoro, v. 24, n. 3, p. 158-166, jul.-set., 2011

SAKAMOTO, A. Y., QUEIROZ-NETO, J. P.; FERNANDES, E.; LUCATI, H. M. Topografia de lagoas salinas e seus entornos no Pantanal de Nhecolândia-MS. In: SIMPÓSIO SOBRE RECURSOS NATURAIS E SÓCIO-ECONÔMICOS DO PANTANAL - MANEJO E CONSERVAÇÃO, 2., 1996. Brasília. Anais... Brasília: EMBRAPA, 1996. v. 1, p. 1-12.

Secretaria de Estado de Meio Ambiente, do Planejamento, da Ciência e Tecnologia e Instituto de Meio Ambiente de Mato Grosso do Sul Plano estadual de recursos hídricos de Mato Grosso do Sul. Campo Grande, MS: Editora UEMS, 194p, 2010.

SORIANO, B.M.A. \& M.J.M. ALVES.. Boletim Agrometeorológico, Ano 2001 para a sub-região da Nhecolândia, Pantanal, Mato Grosso do Sul, EMBRAPA/Pantanal, Corumbá, MS. 28p.,2003.

STIGTER, T.Y.; RIBEIRO, L.; CARVALHO DILL, A.M.M. Application of a groundwater quality index. Journal of Hydrology, Amsterdam, v.327, n.3-4, p.578-591, 2006.

ZAVARZIN, G. A. Microbial geochemical calcium cycle. Microbiologia, v. 71, p. 1-17, 2002.

ZHOU, R.; ZHU, L.; KONG, Q. Persistent chlorinated pesticides in fish species from Qiantang River in East China. Chemosphere, Amsterdam, v.68, n.5, p.838-847, 2007. 